\title{
Presentación atípica de nocardiosis pulmonar como pioneumotórax en un paciente en tratamiento con corticoides
}

\author{
Unusual presentation of pulmonary nocardiosis as pyopneumothorax \\ in a patient under corticotherapy.
}

\begin{abstract}
Nocardiosis is a rare opportunistic infection caused by the bacteria Nocardia spp. It may present as a localized infection or as an invasive infection (pulmonary, central nervous system or disseminated). The authors describe a 76-year-old woman under corticotherapy for the last month because of a recent diagnosis of cryptogenic organising pneumonia. She presented to the emergency department with one-week evolution of pleuritic chest pain and shortness of breath. Chest radiograph showed a left hydropneumothorax. A chest tube was inserted, and the drained pleural effusion was purulent, compatible with a pyopneumothorax. The pleural fluid culture came positive for Nocardia cyriacigeorgica. Nocardiosis is a serious infection with non-specific presentation making its diagnosis a challenge.
\end{abstract}

Keywords: Nocardia, Pneumothorax, Inmunosupression.

Palabras clave: Nocardia, Neumotórax, Inmunosupresión.

\section{HISTORY}

A 76-year-old caucasian woman, with no smoking history, presented to the emergency department with one-week evolution of pleuritic chest pain and shortness of breath. She had medical history of hypertension and stage IV chronic renal disease. For the last year, she complained of fatigue, dry cough and wheezing. High resolution thoracic computed tomography revealed bilateral large areas of ground glass, signs of generalized fibrosis and some emphysematous bullae in the lower inferior lobes. The features of lung biopsy from thoracoscopy were compatible with organizing pneumonia. The study of autoimmunity, HIV1 and 2 serology were negative and there was no identifiable cause for the organizing pneumonia, reason why, she was diagnosed with cryptogenic organizing pneumonia. Therefore, she was under oral prednisolone $60 \mathrm{mg}$ per day) for the last month. At presentation, physical examination was notable for tachypnea, oxygen saturation was $89 \%$ while she was breathing ambient air and lung examination revealed reduced breathing sounds in the left lung field.

\section{INVESTIGATIONS}

Laboratory evaluation was notable for leukocytosis (11300 cells $/ \mathrm{mL}$ ), neutrophilia (97\%) and C-reactive protein of $53 \mathrm{mg} / \mathrm{dL}$. Arterial blood gas test in ambient air revealed hypoxemia $(58 \mathrm{mmHg})$ and chest radiograph showed a left hydropneumothorax. A chest tube was inserted, and the drained pleural effusion was purulent, compatible with a pyopneumothorax in a patient with cryptogenic organizing pneumonia under corticotheraphy. Furthermore, blood and pleural fluid cultures were collected.
Fig. 1. Thoracic CT showing bilateral areas of ground glass opacities, reticulation pattern and traction bronchiectasis.

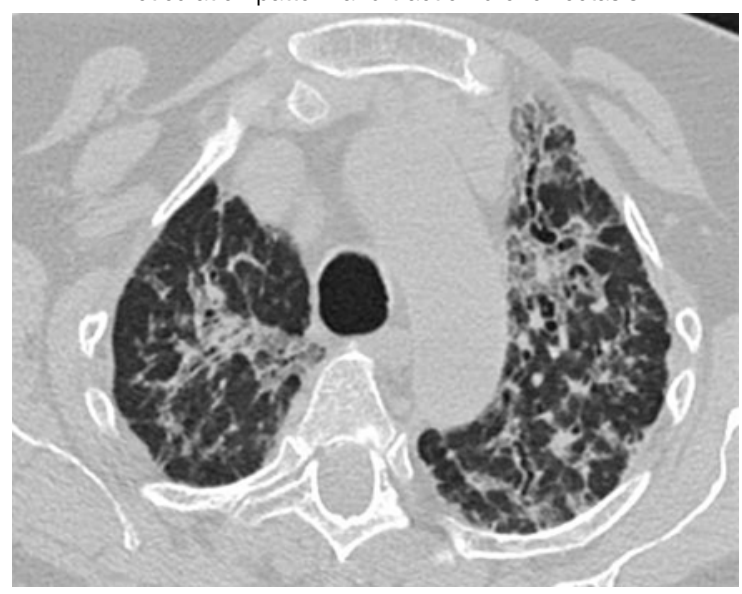

Fig. 2. Thoracic CT showing an extensive left pneumothorax.

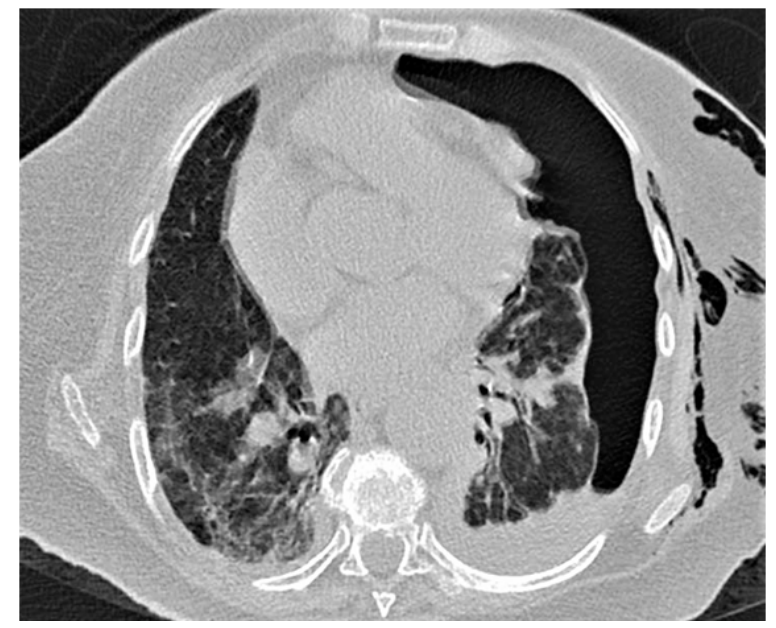

Fig. 3. Chest $X$ ray showing a left hydropneumothorax.

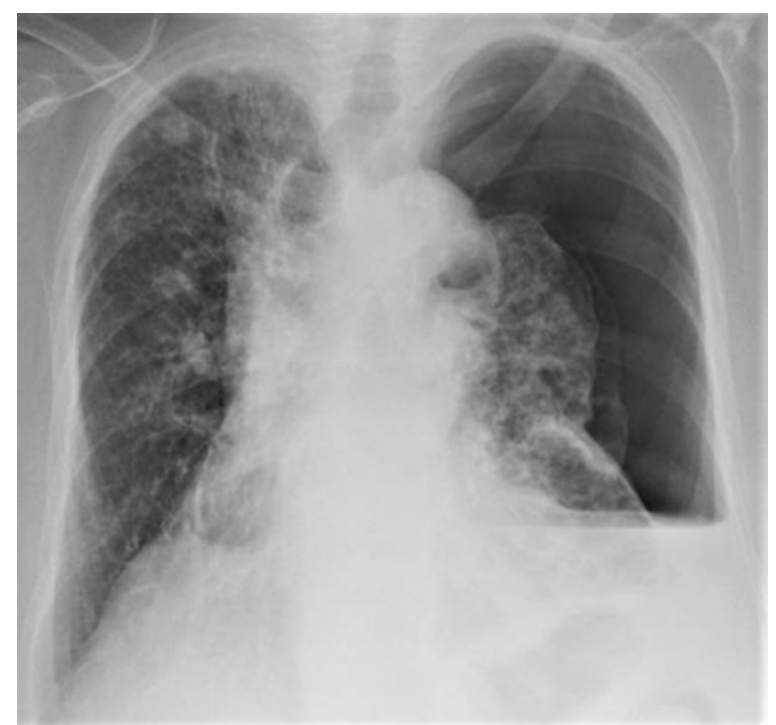




\section{RESULTS AND TREATMENT}

The patient was started on ceftriaxone (2g per day). After one week, the pleural fluid culture came positive for Nocardia cyriacigeorgica. Besides the clinical and laboratory improvement under ceftriaxone, it was added cotrimoxazole (960mg bid). After nine days of ceftriaxone and four days of cotrimoxazole with clinical and laboratory improvement, there was an aggravation of the pneumothorax because of a high flow bronchopleural fistula which aggravated the respiratory insufficiency. An orotracheal tube was inserted and the patient was started on invasive mechanical ventilation and transferred to a critical care unity. Unfortunately, she died after a second, right pneumothorax development.

\section{CONCLUSIONS AND POINTS FOR DISCUSSION}

This clinical case illustrates a rare presentation of a pyopneumothorax caused by Nocardia cyriacigeorgica infection in an immunocompromised host (under systemic corticotherapy and with stage IV chronic renal disease). However, and despite the adequate application of antibiotic therapy when Nocardia cyriacigeorgica was isolated in pleural fluid, the patient's comorbidities, especially chronic renal disease, cryptogenic organizing pneumonia and the difficult to resolve pneumothorax contributed to the fatal outcome. Nocardia spp infection occurs mainly in immunocompromised individuals. Laboratory diagnosis of nocardiosis is difficult considering that Nocardia species require prolonged incubation periods.

André Fabiano, Anatilde Trinidade

Hospital Profesor Doutor Fernando Fonseca 